\title{
Snapshots of neutrino oscillation physics today and a preview of tomorrow
}

\author{
S. Manly ${ }^{1, a}$ \\ on behalf of the T2K collaboration \\ ${ }^{1}$ University of Rochester, Rochester NY, USA 14627
}

\begin{abstract}
Though neutrinos were first observed over 60 years ago, many interesting and fundamental questions remain to be answered. This paper provides a brief overview of neutrino oscillation physics (in the 3-neutrino limit) and how it contributes to our overall understanding of neutrinos. Selected results from current long baseline and reactor experiments are discussed. The increasingly important role played by neutrino interaction physics in oscillation analyses is explored. Finally, the status and goals of selected future experiments are mentioned.
\end{abstract}

\section{Introduction}

Though neutrinos were first observed experimentally over 60 years ago, they continue to be a fertile ground for physics research. These days, a very intense area of research is that of neutrino flavor oscillation physics. Neutrino oscillations were first observed by the Super-Kamiokande experiment in 1998 [1]. They observed a deficit in upward going muon neutrinos formed in cosmic ray interactions in the atmosphere relative to downward going muon neutrinos. Since then, numerous experiments have observed neutrino oscillations in atmospheric neutrinos, solar neutrinos, reactor neutrinos and neutrinos produced in accelerators [2].

In the three neutrino paradigm that is commonly used as a framework for understanding neutrino oscillations, there are three flavors of neutrinos, the electron neutrino $\left(v_{e}\right)$, the muon neutrino $\left(v_{\mu}\right)$, and the tau neutrino $\left(v_{\tau}\right)$. Neutrinos are created or destroyed in states of definite flavor. There are also three neutrino mass eigenstates, $v_{1}, v_{2}$, and $v_{3}$. The flavor eigenstates and mass eigenstates do not have a one-to-one correspondence. The mass eigenstates are related to the flavor states via the PMNS mixing matrix [3]. In the PMNS formalism, there are three mixing angles, $\theta_{13}, \theta_{23}, \theta_{12}$, and one complex phase, $\delta$. The PMNS matrix is commonly shown as below, where it is factorized into three experimental regimes via matrices where each part depends on one mixing angle and where $c_{i j}=\cos \theta_{i j}$ and $s_{i j}=\sin \theta_{i j}$.

$$
U=\left(\begin{array}{ccc}
1 & 0 & 0 \\
0 & c_{23} & s_{23} \\
0 & -s_{23} & c_{23}
\end{array}\right)\left(\begin{array}{ccc}
c_{13} & 0 & s_{13} e^{-i \delta} \\
0 & 1 & 0 \\
-s_{13} e^{i \delta} & 0 & c_{13}
\end{array}\right)\left(\begin{array}{ccc}
c_{12} & s_{12} & 0 \\
-s_{12} & c_{12} & 0 \\
0 & 0 & 1
\end{array}\right)
$$

\footnotetext{
ae-mail: steven.manly@ rochester.edu
} 
Neutrino oscillations are thought to arise when a neutrino formed in a state of definite flavor propagates. The linear combination of mass eigenstates corresponding to the initial flavor state will evolve in time, leading to changes in the mix of neutrino flavors in the beam, i.e., fewer than expected neutrinos of the original flavor or the appearance of flavors not present at inception.

As an illustrative example of this phenomenon, consider a beam of pure muon neutrinos of energy E that propagate a distance L. The probability for neutrinos in this beam to be detected as electron neutrinos, the so-called appearance probability, is given by [4]

$$
\begin{aligned}
P\left(v_{\mu} \rightarrow v_{e}\right) & =4 c_{13}^{2} s_{13}^{2} s_{23}^{2} \sin ^{2} \Delta_{31} \\
& +8 c_{13}^{2} s_{12} s_{13} s_{23}\left(c_{12} c_{23} \cos \delta-s_{12} s_{13} s_{23}\right) \cos \Delta_{32} \sin \Delta_{31} \sin \Delta_{21} \\
& -8 c_{13}^{2} c_{12} c_{23} s_{12} s_{13} s_{23} \sin \delta \sin \Delta_{32} \sin \Delta_{31} \sin \Delta_{21} \\
& +4 s_{12}^{2} c_{13}^{2}\left(c_{12}^{2} c_{23}^{2}+s_{12}^{2} s_{23}^{2} s_{13}^{2}-2 c_{12} c_{23} s_{12} s_{23} s_{13} \cos \delta\right) \sin ^{2} \Delta_{21} \\
& -8 c_{13}^{2} s_{13}^{2} s_{23}^{2} \frac{a L}{4 E_{v}}\left(1-2 s_{13}^{2}\right) \cos \Delta_{32} \sin \Delta_{31} \\
& +8 c_{13}^{2} s_{13}^{2} s_{23}^{2} \frac{a}{\Delta m_{31}^{2}}\left(1-2 s_{13}^{2}\right) \sin ^{2} \Delta_{31},
\end{aligned}
$$

where $\Delta_{i j}=m_{j}^{2}-m_{i}^{2}$ and the matter parameter, $a=2 \sqrt{2} \mathrm{G}_{F} \mathrm{n}_{e} \mathrm{E}_{v}$. For anti-neutrinos, $a \rightarrow-a$ and $\delta \rightarrow-\delta$.

Within this formalism, a number of questions are driving current efforts in long baseline oscillation research.

- What is the mass hierarchy? Oscillation measurements to date have measured a mass splitting between $v_{2}$ and $v_{3}$ to be fairly big, $\sim \pm 2.4 \times 10^{-3} \mathrm{eV}^{2}$, and that between $v_{1}$ and $v_{2}$ to be smaller, $\sim 7.6 \times 10^{-5} \mathrm{eV}^{2}$. What is not known is whether the mass of $v_{3}$ is larger than the masses of $v_{1}$ and $v_{2}$ (a condition known as normal ordering) or if it is smaller than that of $v_{1}$ and $v_{2}$ (known as inverted ordering).

- Is the CP violating phase, $\delta$, non-zero? If so what is it? This gets at whether or not neutrinos and anti-neutrinos have an inherent difference in how they oscillate (beyond that which arises from matter effects).

- Is $\theta_{23}$ maximal? If not, what quadrant is it in, i.e., is it greater than or less than $45^{\circ}$ ? This tells us whether or not the $v_{\mu}$ and $v_{\tau}$ content in mass eigenstate 3 are equal.

In the paper that follows, a few of the experiments and measurements that are of current and nearfuture importance in this area of research are briefly reviewed. This is not an inclusive review. With regret, much relevant and important work is not included here in the interest of brevity.

\section{Reactor experiments}

The most precise determinations of $\sin ^{2} 2 \theta_{13}$ have been done through measurements of the disappearance of electron anti-neutrinos produced by powerful nuclear reactors. These experiments detect the anti-neutrinos through the inverse beta decay process in detectors at different baselines from one or more nuclear reactor cores. Three different experiments have released results recently, Double Chooz in France [5], Reno in Korea [6], and Daya Bay in China [7]. The detectors have baselines that are typically hundreds of meters. As an example of the results achieved in these experiments, Figure 1 shows the survival probability for electron anti-neutrinos as a function of the effective baseline divided by the neutrino energy from a recent publication from the Daya Bay experiment [8]. The 
anti-neutrino disappearance signal is obvious. A recent compilation of measurements of $\sin ^{2} 2 \theta_{13}$ is shown in Figure 2.

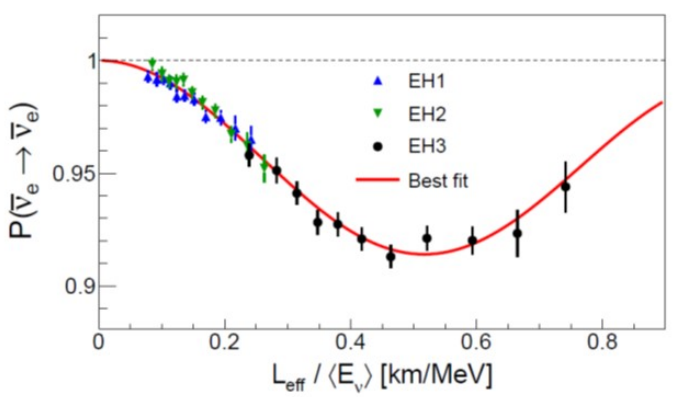

Figure 1. The survival probability for electron anti-neutrinos as a function of effective $\mathrm{L} / \mathrm{E}$ for the Daya Bay experiment, from [8].

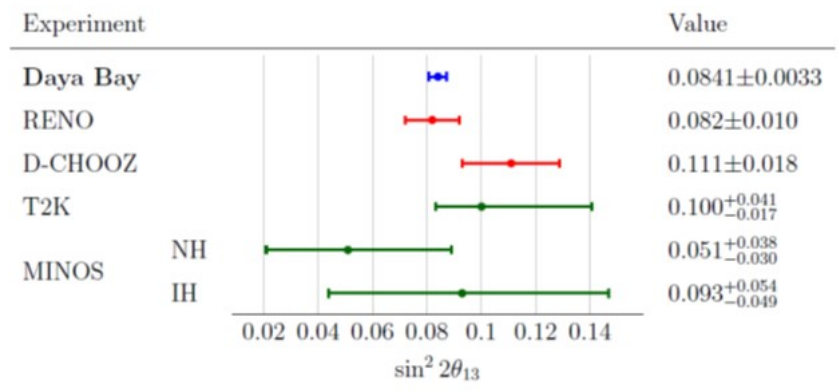

Figure 2. A compilation of recent measurements of $\sin ^{2} 2 \theta_{13}$ from [8].

\section{Long baseline experiments}

Three experiments have released long baseline neutrino oscillation results recently, T2K in Japan [9] and MINOS [10] and NOvA [11] in the United States. All three experiments operate with beams created by decaying, magnetically-focused hadrons produced in proton collisions on a fixed target. Each experiment uses a near detector, located $\leq 1 \mathrm{~km}$ from the target, to constrain the beam simulation, and a far detector some distance away to measure the oscillated neutrino beam. The MINOS far detector is located at a distance of $735 \mathrm{~km}$ on the axis of the neutrino beam. The far detectors for the other two experiments are situated off axis, at $2.5^{\circ}$ for $\mathrm{T} 2 \mathrm{~K}$ and $0.8^{\circ}$ for NOvA, at distances of 295 $\mathrm{km}$ and $810 \mathrm{~km}$ from the target, respectively. The off-axis neutrino flux is relatively narrow in energy spread and lower in energy than the on-axis beam. Each of these experiments has a rich physics program and numerous publications. Only select recent results will be mentioned here.

\subsection{T2K}

During the summer of 2017, T2K released results from analyses done with data taken through the first half of 2017 [12]. The data samples used in this work corresponded to $14.7 \times 10^{20}$ protons-on-target (POT) in the beam configuration optimized to produce neutrinos, i.e., neutrino mode, and $7.6 \times 10^{20}$ POT in anti-neutrino mode. The beam flux and cross section models used in these analyses were constrained with the ND280 near detector, which is made of a mix of time projection chambers and 
plastic scintillator trackers in a magnetic field [9]. The far detector used in the oscillation analyses is a large water Cherenkov detector called Super-Kamiokande [13].

After event selection criteria (described in [12]) were applied, the muon neutrino and anti-neutrino spectra as a function of reconstructed (anti-)neutrino energy were determined, as shown in Figure 3. Note the significant and characteristic dip at $\sim 700 \mathrm{MeV}$ from (anti-)neutrino disappearance due to oscillations. Figure 4 shows the similar spectra for electron (anti-)neutrino appearance. The neutrino energy in both plots in Figure 3 and the two left-most plots in Figure 4 were reconstructed assuming the charged-current quasi-elastic hypothesis. The right plot in Figure 4 is for events that come from charged-current single charged pion production. This latter category is newly added for the analyses released in 2017.
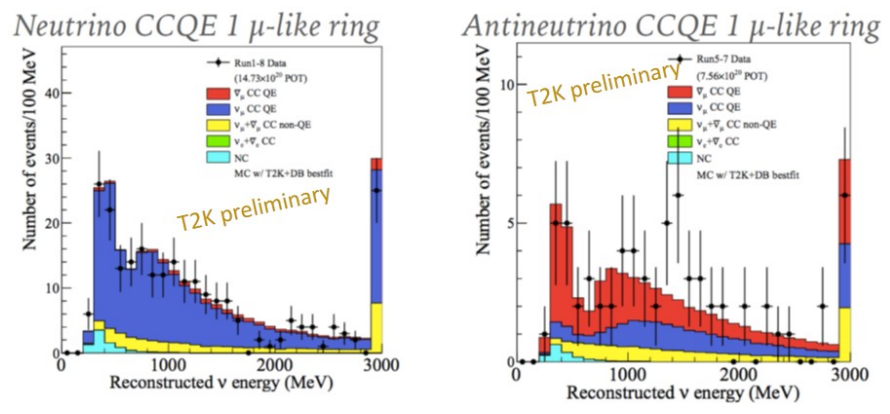

Figure 3. Muon neutrino disappearance spectra for T2K using data taken through mid-2017. The data points are shown in black and the simulated data is shown for the best fit oscillation parameters.
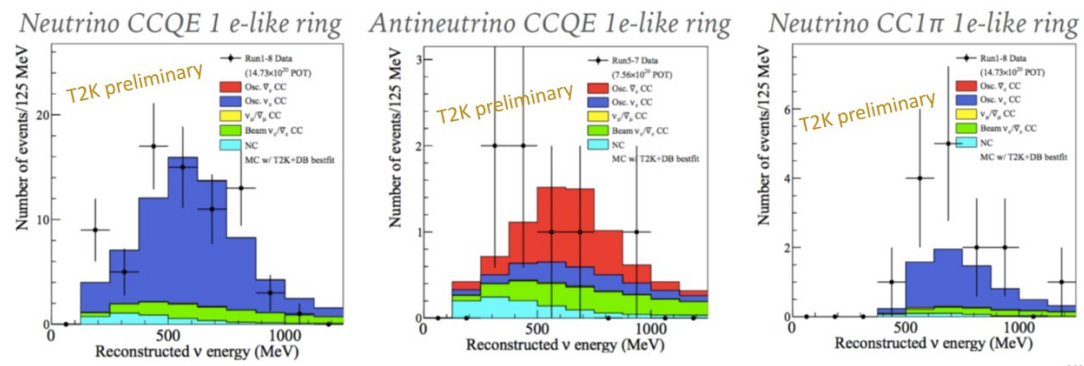

Figure 4. Electron neutrino appearance spectra for $\mathrm{T} 2 \mathrm{~K}$ using data taken through mid-2017. The data points are shown in black and the simulated data is shown for the best fit oscillation parameters.

The total number of observed electron neutrino events (89) is greater than the expectation from oscillations with no $\mathrm{CP}$ violation (67.5). The total number of observed electron anti-neutrinos (7) is less than the expectation from oscillations with no $\mathrm{CP}$ violation (9). These numbers are more consistent with $\delta_{C P}=-\pi / 2$ than with $\delta_{C P}=0$. Figure 5 shows the change in the likelihood as a function of $\delta_{C P}$ for a fit of the data for for cases of both normal hierarchy and inverted hierarchy, where the other parameters in the fit are marginalized over and $\sin ^{2} \theta_{13}$ is taken from reactor experiments. Note that the CP conserving values of 0 and $\pi$ are outside the $2 \sigma$ intervals for both hierarchies.

\subsection{NOvA}

The NOvA experiment began taking data in September of 2013. Since then, they have taken data with both horn polarities. This year they published $v_{\mu}$ disappearance results with $6.05 \times 10^{20}$ POT of 


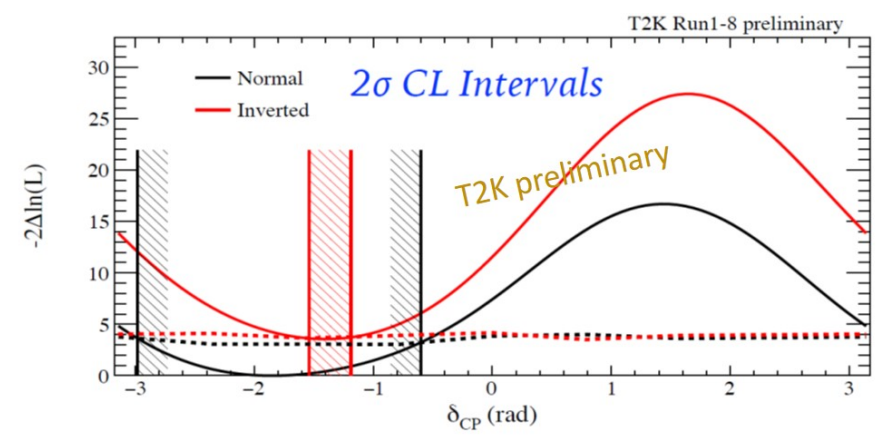

Figure 5. Preliminary results from $\mathrm{T} 2 \mathrm{~K}$ showing the consistency of the data with the oscillation hypothesis as a function of the value of $\delta_{C P}$.

neutrino beam data [14]. As shown in Figure 6, they measured $\sin ^{2} \theta_{23}$ and $\Delta m_{32}^{2}$. They find maximal mixing $\left(\sin ^{2} \theta_{23}=0.5\right)$ is disfavored at $2.6 \sigma$.

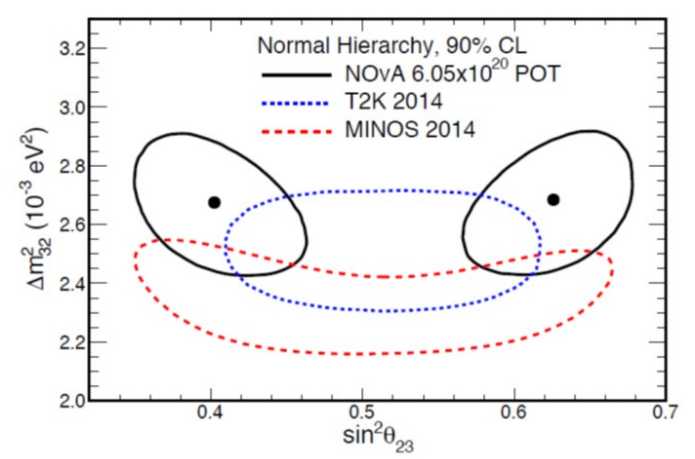

Figure 6. The $90 \%$ C.L. regions for the NOvA best fit in the $\sin ^{2} \theta_{23}-\Delta m_{32}^{2}$ parameter space for normal hierarchy mass ordering overlaid with recent results from T2K and MINOS. From [14].

NOvA has also published results on electron neutrino appearance recently [15]. They observed 33 $v_{e}$ candidate events in the far detector using the same data set as above. Taking the $\theta_{13}$ constraint from reactor experiments and fitting the $v_{\mu}$ and $v_{e}$ spectra simultaneously for $\sin ^{2} \theta_{23}, \delta_{C P}$, and $\Delta m_{32}^{2}$, NOvA finds the results presented in Figure 7. For the hypothesis of inverted mass hierarchy, they disfavor $\theta_{23}$ in the lower octant at greater than $93 \%$ C.L.

\section{Neutrino interactions}

As neutrino oscillation experiments strive to make more precise measurements, neutrino interaction physics becomes more important. It comes into the oscillation analyses in a number of different ways. The accurate reconstruction of the incoming neutrino energy is critical since the oscillation probability and parameter extraction depend on it. To the extent the energy is not reconstructed accurately, the energy resolution and bias must be modeled well, and these depend on the morphology of the event and final state interactions in the nucleus. The neutrino interaction model is also an important part of determining the signal and backgrounds in the far detector. Even though the near detector is used to constrain the neutrino flux in the beam, the fluxes seen at the near detector and the far detector differ. The neutrino interaction model is essential in determining how the expected flux for a given set of oscillation parameters is manifested in the observed far detector neutrino spectra. It is critical for determining the acceptance and backgrounds in the far detector. Through all the effects listed above, errors in the neutrino interaction model lead to a significant source of systematic error in the final oscillation results for long baseline experiments. 

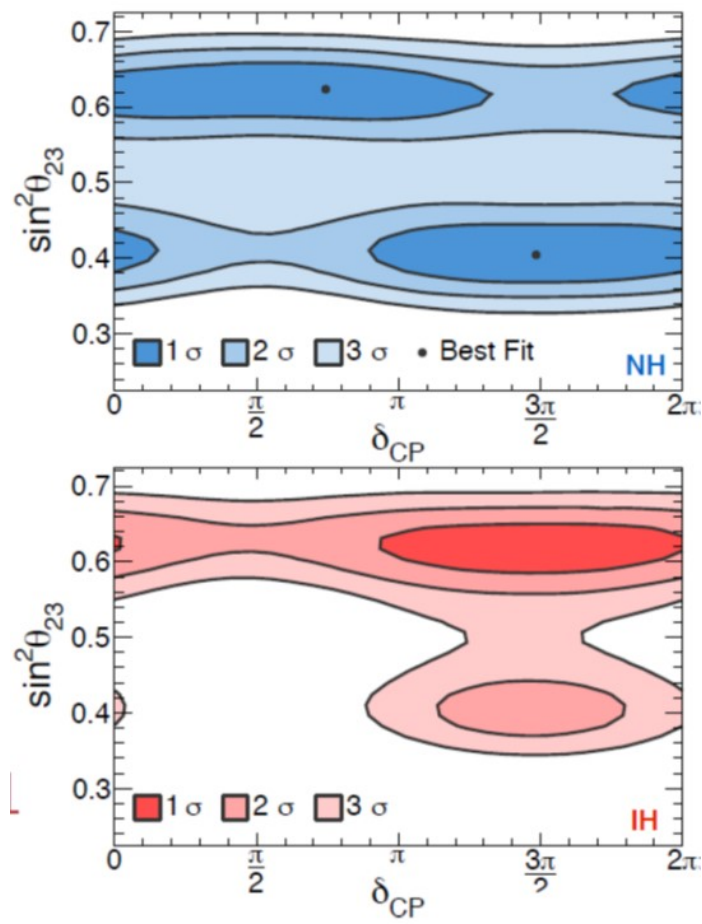

Figure 7. Confidence level intervals for the best fit NOvA results in the $\sin ^{2} \theta_{23}-\delta_{C P}$ plane for normal hierarchy mass ordering on top and inverted hierarchy on bottom. From [15].

In the last couple of years, two experiments in particular have contributed significantly to the understanding and modeling of neutrino interactions: T2K and MINERvA [16]. T2K has used data recorded with the ND280 (near) detector to make numerous cross section measurements on scintillator and water targets [17]. The measurements are of $v_{\mu}$ and $v_{e}$ charged-current interactions as well as neutral-current interactions. The measurements have been made of CCQE interactions (using muon and proton variables) as well as morphologies containing pions. Recently, T2K has shown progress using transverse momentum balance variables to enhance sensitivity to nuclear and final state interactions effects. T2K plans to upgrade the ND280 detector and continue taking data (see Section 5.2 below).

MINERvA is situated $1035 \mathrm{~m}$ from the target in the intense NuMI neutrino beam, just in front of the MINOS near detector (which acts as a muon spectrometer for MINERvA). MINERvA is a fully active tracker made of plastic scintillator with a number of passive nuclear targets (helium, carbon, iron, lead, water) installed on the upstream end. The tracker is surrounded by electromagnetic and hadronic calorimeters. MINERvA has recorded significant data sets with two different wideband, on-axis beam configurations. In the low (medium) energy configuration the neutrino flux peaks at approximately $3.5(6.5) \mathrm{GeV}$. This is somewhat higher than the beam incident on T2K's ND280 detector, providing complementarity and covering the energy range relevant for planned future long baseline neutrino experiments. The MINERvA experiment has released numerous cross section measurements on scintillator and the nuclear targets and continues to take data [18]. 


\section{Near future}

\subsection{JUNO}

The next big step in reactor neutrino oscillation physics is the JUNO experiment in Guangdong Province, China [19]. The JUNO detector will consist of 20 ktons of liquid scintillator. It will be sited with $700 \mathrm{~m}$ of overburden at a distance of slightly over $50 \mathrm{~km}$ from two different powerful nuclear reactor complexes. At this distance, the detector is located at the first oscillation minimum driven by $\theta_{12}$ as shown in Figure 8. The electron anti-neutrino flux as a function of L/E for JUNO is

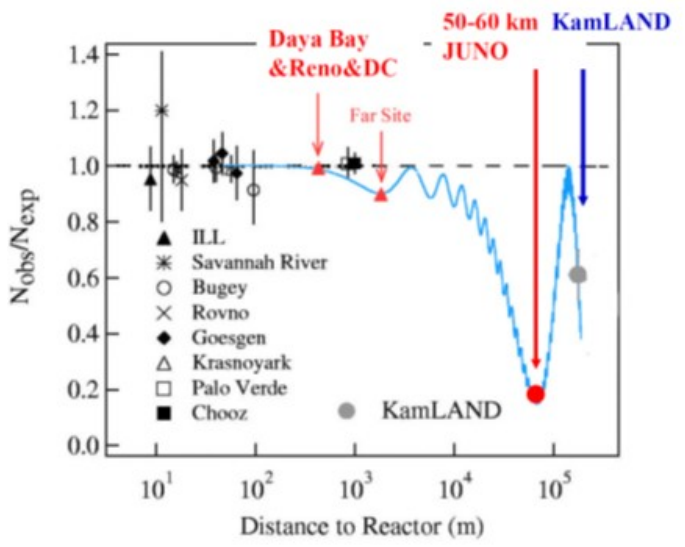

Figure 8. The electron anti-neutrino survival probability as a function of distance to the reactor source for the JUNO experiment. From [20].

shown in Figure 9. There is a small ripple in the flux as a function of $\mathrm{L} / \mathrm{E}$ that depends on the mass hierarchy. This gives JUNO good sensitivity for determining the mass hierarchy of the neutrinos. In addition to neutrino oscillations, JUNO will have a rich program of physics studying supernova neutrinos, geoneutrinos, atmospheric neutrinos and proton decay, as well as searches for other physics. JUNO is under construction and the hope is for the experiment to begin operation in 2020.

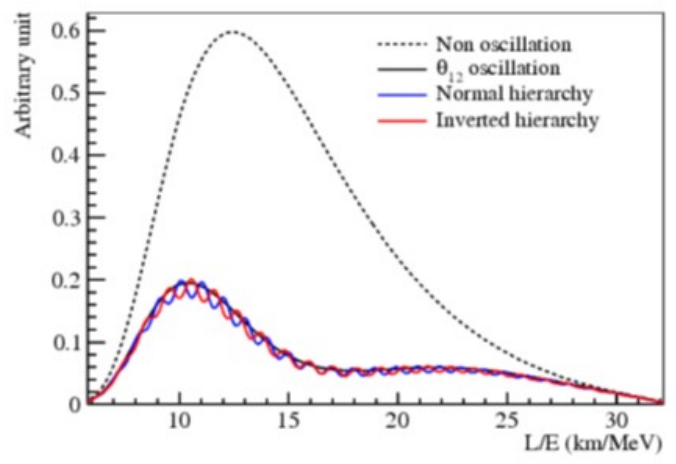

Figure 9. The electron anti-neutrino flux as a function of L/E for the JUNO experiment. From [20].

\subsection{T2K II}

$\mathrm{T} 2 \mathrm{~K}$ has been approved for $7.8 \times 10^{21}$ POT. This is a factor of 3.4 times the data used for the results shown in Section 3.1. The collaboration has requested an extension of T2K operation to 2026 for 
a total POT of $20 \times 10^{21}$, a factor of 9 over the current data set [21]. This extension is known as T2KII. This increase in statistics combined with the expected analysis improvements and detector and operations upgrades will lead to an improvement of $50 \%$ in experimental sensitivity. Figure 10 shows the expected sensitivity of T2KII as a function of $\delta_{C P}$ assuming the currently measured T2K central value reflects the truth. It is possible that T2KII will rule out a non-zero $\delta_{C P}$ at $3 \sigma$ even if the expected improvement in the systematic errors is not realized.

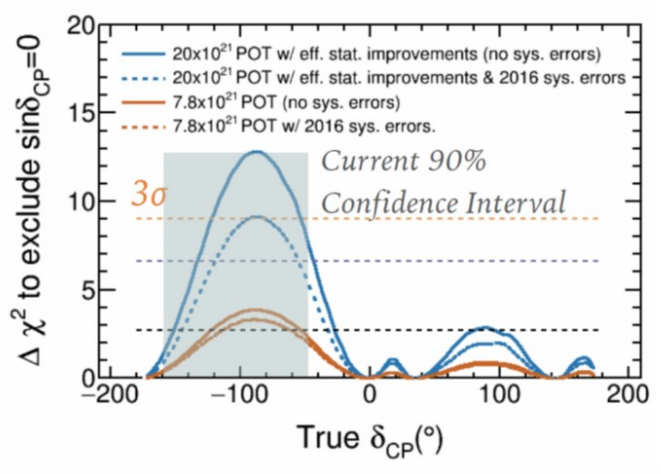

Figure 10. The expected sensitivity of T2KII as a function of $\delta_{C P}$ assuming the currently measured T2K central value reflects the truth. From [12].

\subsection{Hyper-K}

At the end of T2KII, the main ring beam power at J-PARC is projected to level off at approximately $1300 \mathrm{~kW}$ (to date the accelerator has achieved stable operation at $470 \mathrm{~kW}$ ). The HyperKamiokande project is proposed to take data in this beam beginning in 2026. The concept behind Hyper-Kamiokande is to build one, or possibly two, large water Cherenkov detectors, where each has approximately 8 times the fiducial volume as the Super-Kamiokande detector [22]. One detector will be placed near Super-Kamiokande at a baseline of approximately $295 \mathrm{~km}$ from the target and the other, if built, may be placed near the first or possibly in Korea at a baseline of 1000-1300 km. Figure 11 shows the results of a recent sensitivity study for Hyper-Kamiokande [23] in the case of a single tank. In the figure, the significance for the Hyper-Kamiokande experiment to measure CP violation (along with comparisons to other experiments) for the case where $\delta_{C P}=-\pi / 2$ with normal hierarchy mass ordering.

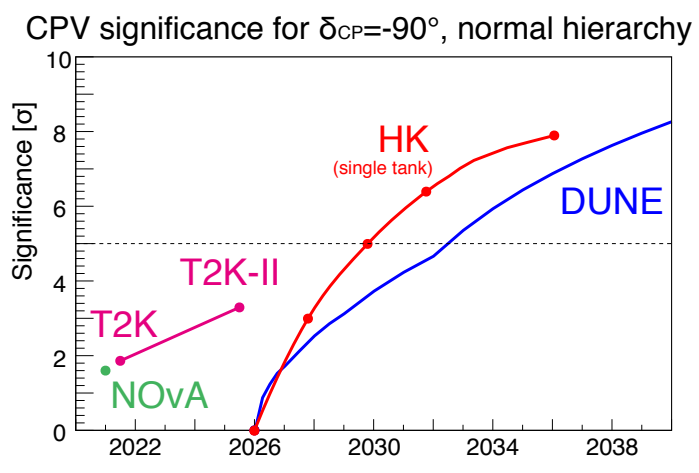

Figure 11. The significance for Hyper-Kamiokande to measure $\mathrm{CP}$ violation for the case where $\delta_{C P}=-\pi / 2$. Projections for other experiments are shown for comparison. From [23]. 


\subsection{DUNE}

The Deep Underground Neutrino Experiment (DUNE) also plans to begin taking neutrino beam data in 2026 [24]. The DUNE far detector will be sited in the Sanford Underground Research Facility in South Dakota, USA. It will consist of four $10 \mathrm{kton}$ liquid argon time projection chambers deployed over time in a staged fashion. The physics program for DUNE is similar to that for HyperKamiokande: long-baseline neutrino oscillations, supernova neutrinos and proton decay. The beam for DUNE will be generated at the Long Baseline Neutrino Facility at Fermilab and pointed directly at the far detector, $1300 \mathrm{~km}$ distant. Figure 12 shows the currently projected sensitivity to $\delta_{C P}$ for DUNE for normal mass ordering [25].

Construction for DUNE is underway. Two large liquid argon prototypes are being built at CERN, with plans to inject test beam into them during the summer and early fall of 2018. Also, recently ground was broken for the large scale underground excavation in South Dakota.

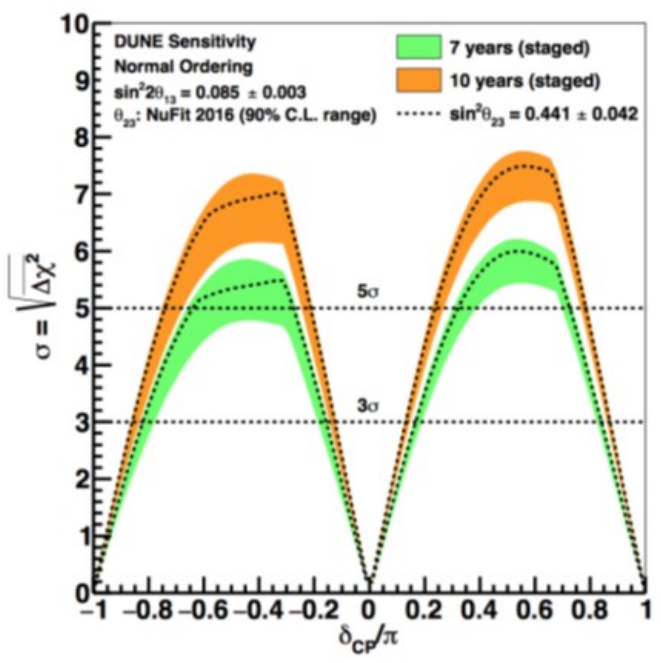

Figure 12. The currently projected sensitivity to $\delta_{C P}$ for DUNE for normal mass ordering.

\section{Conclusion}

Neutrino physics continues to provide fertile ground for discovery. The program of current and planned experiments should provide insights into the potential existence of sterile neutrinos, the degree of CP violation in the neutrino sector, and the mass ordering. Several of these projects will provide the ability to perform proton decay searches with unprecedented sensitivity. With luck, these detectors will also have the opportunity to study neutrinos from a nearby supernova.

\section{References}

[1] Y. Fukuda et al. (Super-Kamiokande Collaboration), Phys. Rev. Lett. 81, 1562-1567 (1998).

[2] G.J. Feldman, J. Hartnell, T Kobayashi, Advances in High Energy Physics 2013, 475749 (2013).

[3] Z. Maki, M. Nakagawa, S. Sakata, Prog. Theoretical Physics 28, 870 (1962) and B. Pontecorvo, Soviet Physics JEPTP 7, 172(1957).

[4] Y. Hayato, presented at Advances in Neutrino Technology 2011, Philadelphia, PA, 2001. See slides at https://indico.fnal.gov/event/4887/session/0/contribution/1/material/slides/0.pdf. 
[5] See the Double Chooz website at http://doublechooz.in2p3.fr/Status_and_News/status_and_ news.php.

[6] RENO experiment, arXiv:1003.1391 (2010).

[7] See the Daya Bay experiment website at http://dayabay.ihep.ac.cn/twiki/bin/view/Public/.

[8] F.P. An et al. (Daya Bay Collaboration), Phys. Rev. D 95, 072006 (2017).

[9] K.Abe et al. (T2K Collaboration), Nucl. Instrum. Meth. A 659, 106-135 (2011).

[10] D.G. Michael et al. (MINOS Collaboration), Nucl. Instrum. Meth. A 596, 190-228 (2008).

[11] See the NOvA experiment website at https://www-nova.fnal.gov/.

[12] M. Hartz (representing the T2K Collaboration), presented at KEK, Tsukuba, Japan, August 2017. See slides at https://t2k.org/docs/talk/282/kekseminar20170804.

[13] S. Fukuda et al. (Super-Kamiokande Collaboration), Nucl. Instru. Meth. A 501, 418-462 (2003).

[14] P. Adamson et al. (NOvA Collaboration), Phys. Rev. Lett. 118, 151802 (2017).

[15] P. Adamson et al. (NOvA Collaboration), Phys. Rev. Lett. 118, 231801 (2017).

[16] L. Aliaga et al. (MINERvA Collaboration), Nucl. Instrum. and Meth. A743, 130 (2014).

[17] See, for example, K. Abe et al. (T2K Collaboration), Phys. Rev. D95, 012010 (2017). Many other relevant T2K publications are listed at https://www.t2k.org/docs/pub.

[18] See, for example, M. Betancourt et al. (MINERvA Collaboration), Phys. Rev. Lett. 119, 082001 (2017). Many other relevant MINERvA publications are listed at http://minerva.fnal.gov/ recent-minerva-results/.

[19] See the JUNO experiment website at http://juno.ihep.cas.cn/.

[20] G. Ranucci, presented at INR, Troisk, Russia, September 10, 2015. See http://www.inr.ru/ $\sim$ pantuev/seminars/JUNO-Troitsk.pdf.

[21] K. Abe et al. (T2K Collaboration), arXiv:1609.04111 [hep-ex], 2016.

[22] K. Abe et al. (Hyper-Kamiokande Collaboration), aXiv:1611.06118 [hep-ex], 2016.

[23] M. Kuze for the Hyper-Kamiokande Proto-collaboration, presented at the PANIC 2017 conference, Bejing, 2017. See slides at http://indico.ihep.ac.cn/event/6329/session/2/contribution/203/ material/slides/0.pdf

[24] See the DUNE experiment website at http://www.dunescience.org/.

[25] From DUNE approved plot archive. 